\title{
Exceso de morbilidad respiratoria en niños y adultos mayores en una comuna de Santiago con alta contaminación atmosférica por partículas
}

\author{
María José Prieto $C^{1 a}$, Pedro Mancilla $F^{1 a}$, Pedro Astudillo $0^{1}$, \\ Álvaro Reyes $\mathrm{P}^{2 \mathrm{~b}}, 0$ scar Román $\mathrm{A}^{\mathbf{1}}$. \\ Excess respiratory diseases in children \\ and elderly people in a community \\ of Santiago with high particulate air \\ pollution
}

Background: The relationship between air pollution and health damage has been sufficiently documented. In station " $\mathrm{R}$ " of the air quality monitoring system, located in a community of Metropolitan Santiago (Cerro Navia), the Chilean standard of $150 \mu \mathrm{g} / \mathrm{m}^{3}$, averaged in 24 hours, for particles with a diameter of 10 micrometers or less $\left(\mathrm{PM}_{10}\right)$, has been exceeded more days than in the rest of the city stations. Aim: To investigate if the population living near that station has a higher proportion of lower respiratory infections than the Metropolitan Region (MR) as a whole. Material and methods: An outpatient clinic located near station " $\mathrm{R}$ " (Centro Albertz), was implemented as a sentinel center according to UNICEF methodology, used since 1992 by the Acute Respiratory Infections National Program. Daily information was collected between May and December 2004. Monitoring data included total number of consults by children less than 15 years old for lower respiratory tract infections, pneumonia, obstructive bronchitis syndrome in children and by adults over 64 years old for lower airway disease, chronic obstructive pulmonary disease (COPD), and pneumonia. Results were compared with those of the rest of MR Results: Compared with the MR, children from the sentinel clinic had a significantly higher proportion of consults for obstructive bronchial syndrome $(20.1 \%$ and $26.4 \%$ respectively, in $p<0.01$ ) and pneumonia (1.3 and $2.7 \%$ respectively, $p \varangle 0.01$ ). In the elderly, the average consults for lower airway disease were $17 \%$ in the sentinel clinic and $12.2 \%$ in $\mathrm{MR}(\mathrm{p} \varangle 0.04)$. Conclusions: Children and elderly subjects at the sentinel clinic had a significantly higher proportion of respiratory infections (pneumonia and obstructive bronchial syndrome in children and lower airway disease in the elderly) as compared to the Metropolitan Region (Rev Méd Chile 2007; 135: 221-28).

(Key words: Air pollution; Bronchial diseases; Lung diseases)

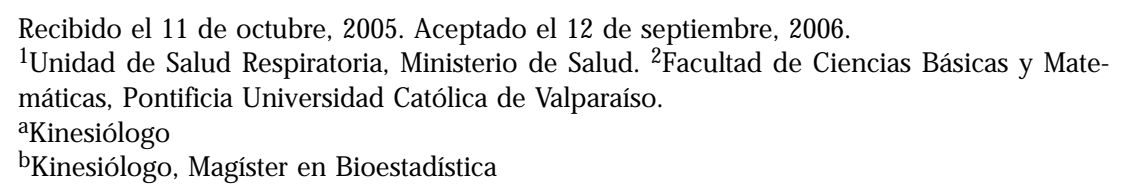

Correspondencia a: Pedro Mancilla F. McIver 459, piso 9, Santiago. Fonos: 5740660-5740699. E mail: programaira@minsal.cl 
E xiste evidencia del daño en salud que produce la contaminación del aire ${ }^{1-8}$. En Estados Unidos de Norteamérica (EE.UU.) y Europa se han constituido centros de estudio de los efectos en salud del material particulado y otros contaminantes. En Chile, se ha comunicado la relación entre las concentraciones de material particulado y sus efectos sobre la morbimortalidad desde la década 1990-992-6.

Los efectos de los contaminantes sobre la salud se dividen en: a) agudos, como irritación de ojos y nariz, aumento de las infecciones respiratorias agudas (IRA) altas y bajas, exacerbaciones del asma bronquial, de enfermedad pulmonar obstructiva crónica (EPOC), de cardiopatías y aumento de las tasas de mortalidad ${ }^{2,6}$ y b) efectos crónicos o diferidos, como el cáncer pulmonar, que es el más representativo ${ }^{7,8}$.

Para medir los efectos en la salud de los contaminantes atmosféricos, es esencial monitorizar, por un lado, la morbilidad, función que se realiza en la Región Metropolitana (RM) desde
1992 a través de Centros Centinela ${ }^{9,10}$ y, por otro, conocer los niveles de los contaminantes atmosféricos, para lo cual opera desde 1987 la Red de Monitoreo Automático de Calidad del Aire (MA$\mathrm{CAM}^{11}{ }^{11}$, bajo responsabilidad del Ministerio de Salud.

Se conoce que la distribución de la contaminación por material particulado de $\mathrm{MP}_{10}$ no es homogénea en las diversas zonas de la Región Metropolitana. Así, la estación de Cerro Navia, denominada estación 〈R», que comenzó su funcionamiento en 2001 y está ubicada al poniente de la ciudad, supera en días con exceso de partículas sobre la norma $\left(150 \mu \mathrm{g} / \mathrm{m}^{3}\right)$ entre 2001 y $2004^{12}$ a todas las demás estaciones de la red. Esto ocurre, no sólo en el número de días en que se sobrepasa la norma de $\mathrm{MP}_{10}$ (Figura 1), sino además en los niveles horarios de concentración de material particulado. Dicha estación cumple con la misma normativa de control técnico que el resto de la Red MACAM, pero no se considera en el sistema de estaciones oficiales que forman parte

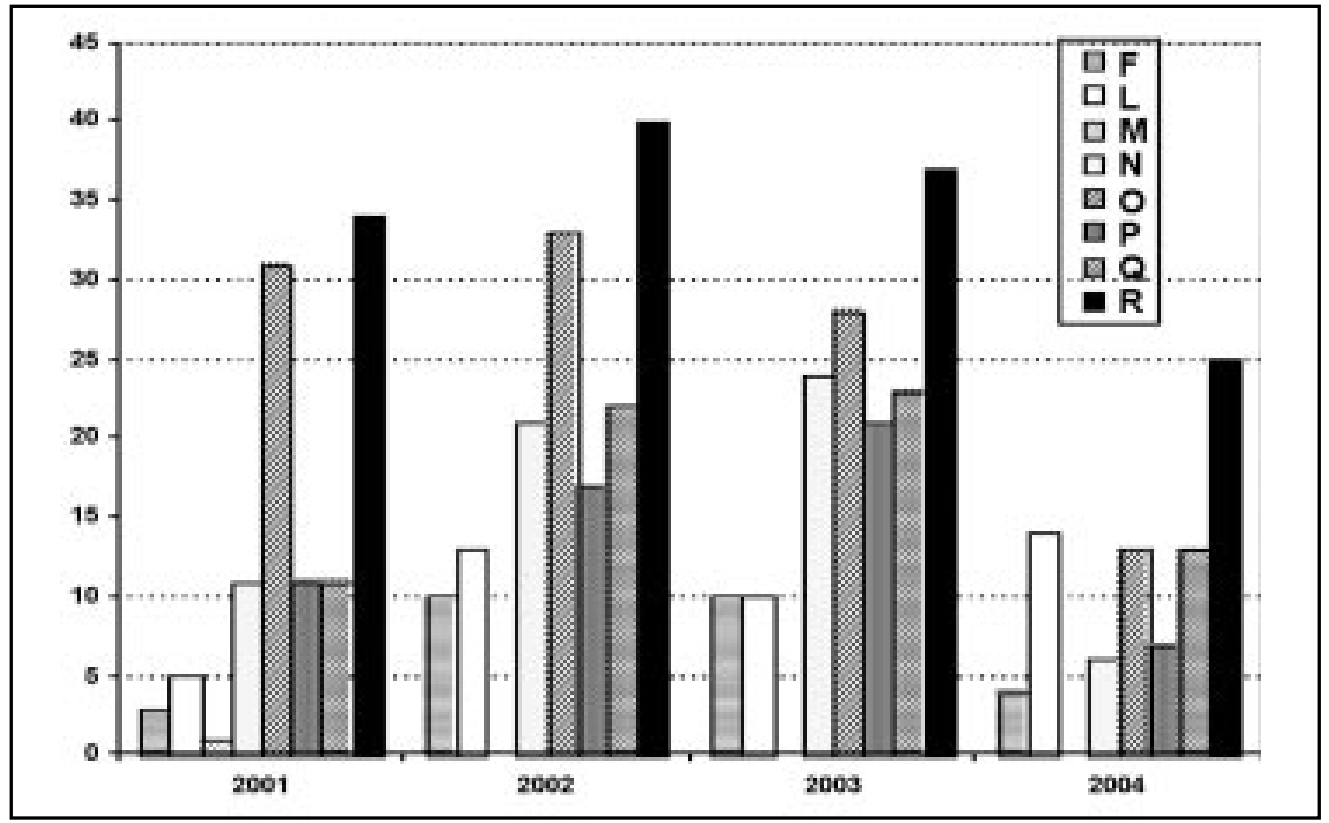

Figura 1. Días por año con promedio diario sobre norma de $\mathrm{MP}_{10}\left(150 \mu \mathrm{g} / \mathrm{m}^{3}\right.$ promedio $\left.24 \mathrm{~h}\right)$ en estaciones de red MACAM y estación «R»de Cerro Navia.

La Figura 1 muestra los días de superación de norma de $\mathrm{MP}_{10}$ de $150 \mu \mathrm{g} / \mathrm{m}^{3}$ en $24 \mathrm{~h}$ por cada año en estaciones de Red MACAM F= Independencia L= La Florida M= Las Condes, N= Santiago $\mathrm{O}=$ Pudahuel, $\mathrm{P}=$ Cerrillos, $\mathrm{Q}=\mathrm{El}$ Bosque y $\mathrm{R}=$ Cerro Navia.

La Estación R, ubicada en Cerro Navia comienza su funcionamiento en 2001, supera los días de excedencia de norma a todas las estaciones entre 2001 y 2004. 
del modelo predictivo para la decisión de contingencias ambientales en la cuenca de la RM.

La demostrada asociación entre contaminación atmosférica por $\mathrm{MP}_{10} \mathrm{y}$ aumento de consultas por causa respiratoria hace suponer que en la población de la comuna de Cerro Navia, la morbilidad respiratoria debeńa ser mayor que en el resto de la RM.

El objetivo de este estudio ha sido verificar si la proporción y severidad de las consultas por enfermedades respiratorias en los consultantes de Centro de Salud Albertz (CA), de la comuna de Cerro Navia, son mayores que en el resto de la RM.

\section{MATERIAL Y MÉTODO}

Según especificaciones de UNICEF9 ${ }^{9}$, se implementó un centro centinela, en el Consultorio Albertz, de la comuna de Cerro Navia, ubicado próximo a la estación «R»de monitoreo de calidad del aire. Este centro llevó a cabo la vigilancia epidemiológica de las enfermedades respiratorias de niños y adultos, registrándose de lunes a viernes el total de consultas de morbilidad, aquéllas por IRA altas y bajas, síndrome bronquial obstructivo (SBO) y neumonía en niños menores de 15 años; los diagnósticos en niños están descritos en la norma del Programa de Infecciones Respiratorias Agudas (IRA) de Chile ${ }^{13}$, utilizada en Atención Primaria. Las clasificaciones IRA alta y baja fueron publicadas en 1998 en serie de Organización Panamericana de Salud ${ }^{9}$. En los adultos de 65 y más años, se registró el total de consultas, aquéllas por ERVAI (que considera enfermedades agudas y crónicas de vía aérea inferior) además de neumonía y EPOC por separado y fueron expresadas como la proporción existente entre la patología respiratonia específica en agrupaciones diagnósticas y el total de consultas de morbilidad general. Los diagnósticos en adultos están descritos en las normas nacionales del Programa de Enfermedades Respiratorias del Adulto $(E R A)^{14}$. En ambos grupos etarios se utilizaron formularios de registro estandanizados para las clasificaciones por diagnósticos. Con el fin de vigilar la oferta de atención, se registró además diariamente la cantidad de horas médicas disponibles.

Los datos obtenidos fueron comparados con los de los demás Centros Centinela de la RM, información proporcionada por el sistema de vigilancia epidemiológica de los programas IRA ${ }^{10}$ y ERA del Ministerio de Salud.
El estudio se realizó desde la semana epidemiológica 20, iniciada el 16 de mayo de 2004, hasta la semana 52, que finaliza el 31 de diciembre del mismo año, completando 31 semanas de vigilancia. Los datos fueron recolectados en el nivel local, pero digitados y analizados en la Unidad de Salud Respiratoria del Ministerio de Salud. Los detalles y la calidad de la información fueron corregidos en visitas de supervisión con pautas estandarizadas y en reuniones periódicas locales.

Se analizaron un total de 92.427 consultas, 12.633 del CA y 79.764 del resto de la RM. Los datos se agruparon según semana epidemiológica, y se expresan como proporción dentro del total de consultas, comparándose las medianas de consultas de cada una de las patologías entre el CA y el resto de la RM. Dado que los datos no se comportaron como una distribución normal, su análisis estadístico se realizó con dócima no paramétrica de suma de rangos con signos de Wilcoxon y se consideró significativo un $\mathrm{p}<0,05$.

\section{RESULTADOS}

En menores de 15 años: Las enfermedades respiratorias agudas infantiles tienen un claro componente estacional, con mayor proporción de consultas por dichas causas en las semanas invernales, distribución que sigue un patrón común en todas ellas. Neumonía se presenta gráficamente con más variación de sus valores de una semana a otra con relación a las demás categorías mórbidas en todo el período observado, pues se expresa como proporción y sus valores absolutos son más pequeños.

IRA baja: no se encontraron diferencias significativas en la mediana de las consultas entre Centro Albertz y RM (Tabla 1).

SBO: la mediana de consultas por esta causa en $\mathrm{CA}$ es significativamente mayor que en la RM (Figura 2).

Neumonía: la mediana de consultas por neumonía es significativamente mayor en CA que en RM (Figura 3).

En adultos de 65 años y más: Las enfermedades respiratorias en el adulto también presentan un componente estacional con mayor proporción de 
Tabla 1. C onsultas por enfermedades respiratorias pediátricas registradas por centros centinela; comparación entre C entro Albertz y Región M etropolitana 2004

\begin{tabular}{|lccc|}
\hline & Centro Albertz & RM & P \\
\hline Mediana IRA baja & 28,66 & 28,48 & 0,60 (NS) \\
Mediana SBO & 26,45 & 20,11 & 0,0001 \\
Mediana neumonía & 2,7 & 1,34 & 0,00001 \\
\hline
\end{tabular}

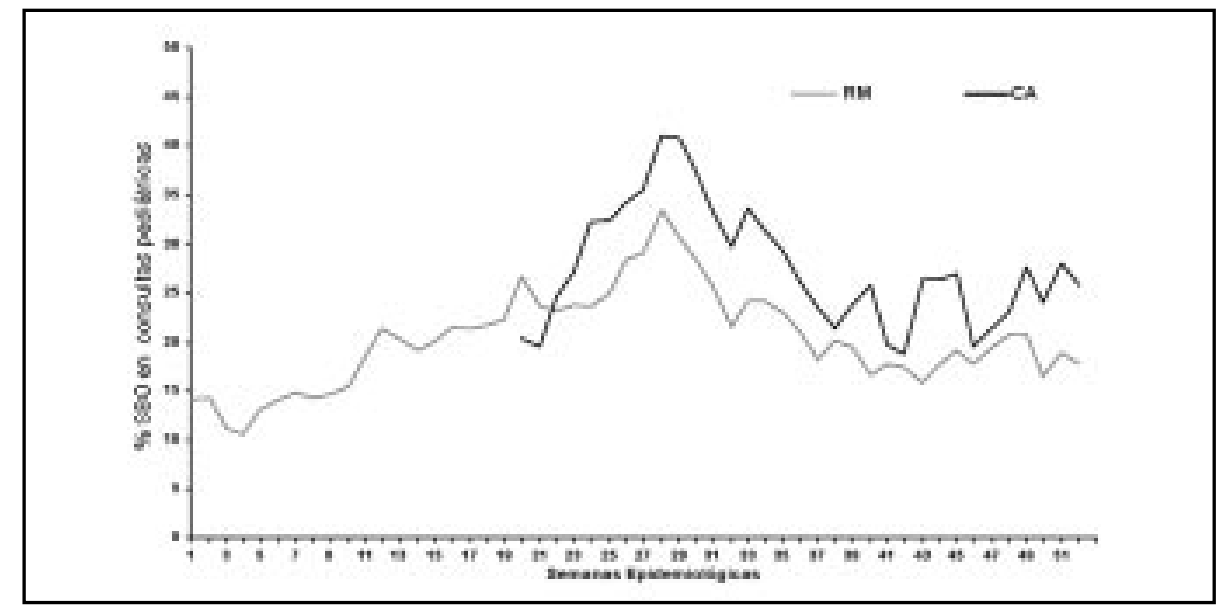

Figura 2. Consultas por síndrome bronquial obstructivo en niños, Región Metropolitana y Centro Albertz.

La Figura 2 muestra las consultas por SBO de niños menores de 15 años expresadas en porcentaje del total de las consultas pediátricas en Centro Albertz (CA) y en los centros centinela de la Región Metropolitana durante año 2004. CA tiene una mayor proporción de consultas por SBO con relación a la mediana de la $\mathrm{RM}(\mathrm{p}<0,0001)$. Los valores de CA se registraron desde semana 20 a la 52.

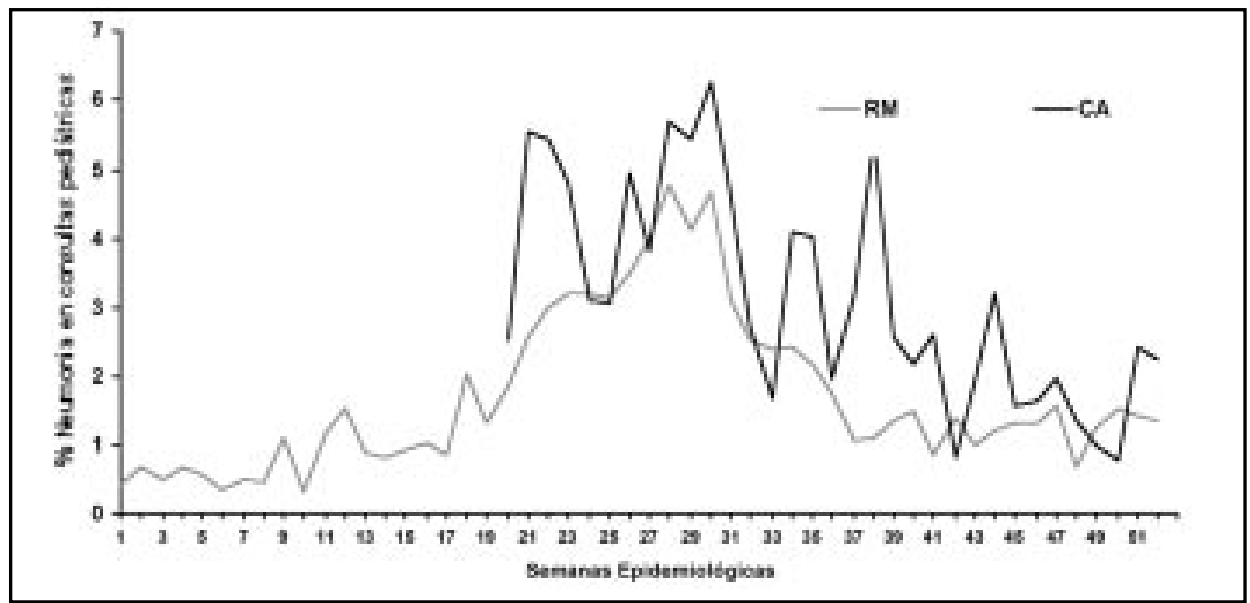

Figura 3. Consultas por neumonía en niños, Región Metropolitana y Centro Albertz.

La Figura 3 muestra las consultas por neumonía de menores de 15 años expresadas como porcentaje del total de las consultas pediátricas en Centro Albertz y en la Región Metropolitana durante año 2004. Centro Albertz tiene una mayor proporción de consultas por neumonía con relación a la mediana de la $\mathrm{RM}$ ( $p<0,00001)$. 
Tabla 2. C onsultas por enfermedades respiratorias del adulto mayor registradas por centros centinela; comparación entre Centro Albertz y Región M etropolitana 2004

\begin{tabular}{|lcrr|}
\hline & Centro Albertz & RM & P \\
\hline Mediana ERVAI & 17 & 12,2 & 0,04 \\
Mediana neumonía & 2,05 & 1,35 & 0,3 (NS) \\
Mediana EPOC & 3,5 & 4,0 & 0,1 (NS) \\
\hline
\end{tabular}

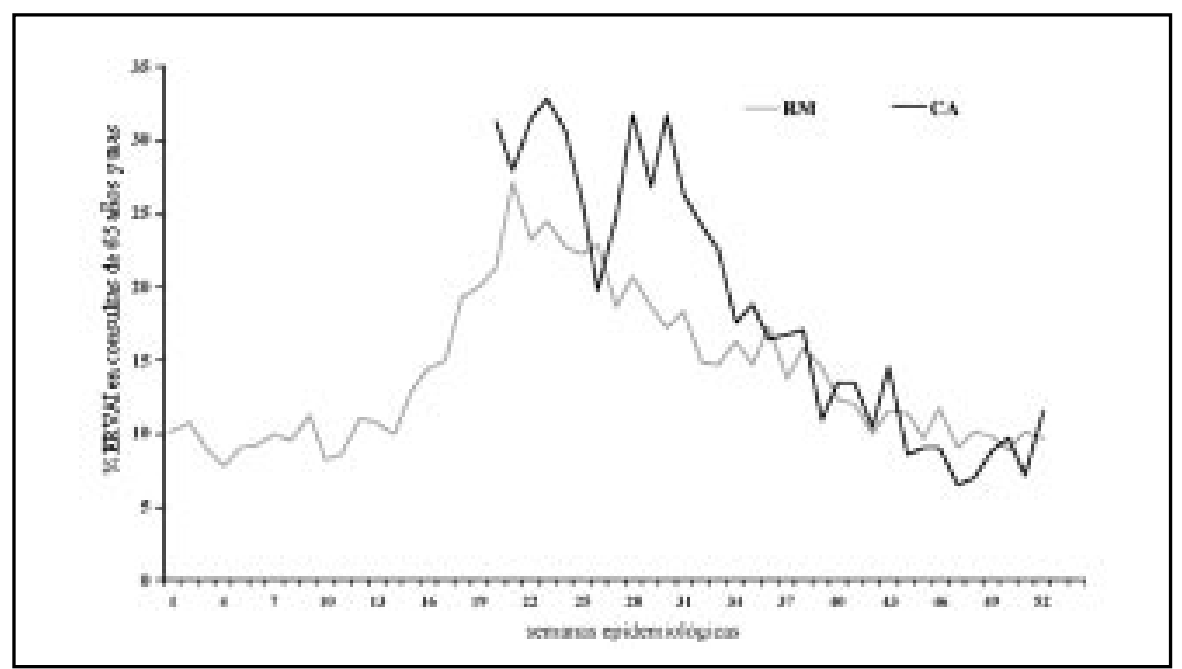

Figura 4. Consultas por enfermedades respiratorias de vía aérea inferior en adultos de 65 y más años, Región Metropolitana y Centro Albertz.

La Figura 4 muestra las consultas por ERVAI de adultos de 65 y más años expresadas en porcentaje del total de las consultas del adulto mayor en Centro Albertz y en la Región Metropolitana durante año 2004. Centro Albertz tiene una significativa mayor proporción de consultas por ERVAI con relación a la mediana de la $\mathrm{RM}(\mathrm{p}<0,04)$.

consultas por dichas causas en las semanas invernales, a excepción de EPOC.

ERVAI: La enfermedad respiratoria de la vía aérea inferior en adultos mayores, fue significativamente mayor en CA que en la RM (Tabla 2 y Figura 4).

Neumonía: No se encontraron diferencias significativas entre CA y Región Metropolitana (Tabla 2).

EPOC: Tampoco se encontraron diferencias por consultas entre RM y el CA (Tabla 2).

\section{DisCUSIÓN}

El Programa Nacional de IRA, realiza en la RM desde 1992, vigilancia de las enfermedades respi- ratorias infantiles a través de Centros Centinela en Atención Primaria ${ }^{10}$, implementados con metodología UNICEF ${ }^{9}$. En adultos, la misma metodología se utiliza desde el año 2002, lo que ha permitido contar con datos confiables para la vigilancia epidemiológica y que estuvieron disponibles para nuestra investigación.

En 1999, Ostro y col$^{3}$ utilizaron la base de datos antes mencionada, de consultas pediátricas en Atención Primaria, conduciendo una investigación con análisis de series de tiempo y regresión múltiple en niños entre 2 y 14 años y en menores de 2 años, encontrando una asociación estadísticamente significativa entre $\mathrm{PM}_{10}$ y consultas por IRA bajas en niños en ambos grupos etarios. En niños 
menores de 2 años, un cambio de $50 \mu \mathrm{g} / \mathrm{m}^{3}$ en $\mathrm{PM}_{10}$ se asoció con un aumento de $4 \%$ a $12 \%$ de consultas por IRA baja; en niños de 2 a 14 años, este aumento osciló entre 3\% y 9\%. También se registró aumento de $5 \%$ en las consultas por IRA baja por cada 50 partes por billón (ppb) de cambio en concentraciones de ozono. Otro estudio hecho en Chile $^{4}$, demostró que durante los meses fríos un aumento de $45 \mu \mathrm{g} / \mathrm{m}^{3}$ en el promedio de $24 \mathrm{~h}$ de material particulado menor de 2.5 micrones $\left(\mathrm{PM}_{2.5}\right)$, se relacionaba con $2,7 \%$ de aumento en el número de consultas pediátricas por causa respiratoria en servicio de urgencia y un aumento de $6,7 \%$ en el número de consultas por neumonía con tres días de rezago, concluyendo que las mezclas de contaminantes urbanos, especialmente el material particulado fino afecta en forma adversa la salud de los niños residentes en Santiago. Con respecto a la asociación entre concentración de contaminantes y morbilidad respiratoria en estudios poblacionales utilizando monitores ambientales, se ha argumentado que la relación patogénica debería relacionarse preferentemente con el ambiente más próximo de cada sujeto. Por ello, investigadores en Chile han realizado mediciones de contaminación intramuros y han encontrado valores más altos de $\mathrm{MP}_{10}$ que los de ambientes exteriores ${ }^{15}$, por lo que la contaminación medida por monitores ambientales a lo menos minimiza la exposición real a la que está sometido el sujeto. Esta observación podría poner en duda la confiabilidad de la correlación entre mediciones de $\mathrm{MP}_{10}$ ambiental y morbilidad observada, sin embargo, estudios epidemiológicos recientes han descartado una asociación por azar o no confiable entre los períodos de mayor concentración de $\mathrm{MP}_{10}$ y morbilidad respiratoria. Además, ha sido mayor el riesgo de la exposición a material particulado fino $\left(\mathrm{MP}_{2.5}\right)$, especialmente en adultos mayores ${ }^{3,8}$.

Las condiciones de la topografía y clima de la cuenca de la Región Metropolitana, zona rodeada de cerros, con escasas lluvias y regímenes de vientos de baja velocidad, con predominio de altas presiones y presencia de inversión térmica, deberían influenciar de manera similar a las distintas comunas en cuanto a contaminación por $\mathrm{MP}_{10}$. Sin embargo, ellas se encuentran a diferentes altitudes, con espacios de diversa cobertura vegetacional, diferente circulación vial, todas va- riables que influyen en la exposición de la población a los contaminantes. Efectivamente, estudios previos han demostrado que la zona poniente de la ciudad de Santiago ${ }^{12}$, presenta mayor número de episodios con concentraciones diarias que superan la norma de $\mathrm{MP}_{10}$. Por otro lado, los centros centinela tienen un comportamiento particular a través de los años, presentando proporciones de morbilidad respiratoria que difieren entre ellos, al igual que la distribución de los contaminantes en la $\mathrm{RM}^{16}$.

Conocidos estos hechos, elegimos el CA de la comuna de Cerro Navia, para establecer la carga de enfermedad de la población consultante. Encontramos que al comparar el CA y el resto de $\mathrm{RM}$, tal como se hipotetizó, las consultas por causa respiratoria a través de las semanas de observación, fueron mayores en el CA de Cerro Navia. Si bien las diferencias no fueron significativas para todas las patologías estudiadas, se evidenció incremento de la proporción en morbilidad respiratoria pediátrica más severa, como SBO y neumonía en los niños, así como enfermedad de vía aérea inferior, en adultos mayores. En los registros de CA no se observó en EPOC el aumento estacional esperado, sino que se mantuvo constante en las semanas de estudio, situación que también se observa en la RM. Esto se debe probablemente a que los médicos de atención primaria registran las exacerbaciones de EPOC como episodio agudo, soslayándose la condición crónica del paciente, con lo cual queda subvalorada la estacionalidad.

La susceptibilidad de enfermar por afecciones respiratorias depende, entre otros factores, del nivel de protección sanitaria de la población, su condición de pobreza, educación y acceso a los servicios de salud ${ }^{17}$. Si bien, según la encuesta CASEN $^{18}$, la pobreza bajó en la RM de 16,1\% en el año 2000 a 13,5\% en el 2003, este descenso no fue homogéneo en toda la región, pues 13 comunas aumentaron su nivel de pobreza. El referido descenso, sí ocurrió en la comuna de Cerro Navia, por lo cual se podría inferir que la mayor magnitud de enfermedad encontrada por nosotros no se debería a un desmedro socioeconómico de esta población, ni tampoco a las condiciones de atención de salud, como disponibilidad de horas médicas y acceso a medicamentos, pues fueron adecuadas para la demanda asistencial en el periodo de estudio. 
Entonces, esta mayor proporción y severidad de las enfermedades respiratorias observadas en CA podría deberse a que en el período otoñoinvierno, las partículas se concentran especialmente en la zona centro y poniente de la cuenca $^{19}$, es decir, pueden estar asociadas a un riesgo ambiental por contaminación del aire.

Es preciso comentar que el año 2004 fue un año epidémico por influenza y VRS que afectó a ambas poblaciones estudiadas y a todo el país ${ }^{20}$.

En este contexto, es necesario recomendar, un estudio más a fondo de los factores asociados al aumento de la morbilidad respiratoria, sin perjuicio de lo cual, esta investigación sugiere la necesidad de establecer medidas preventivas, tanto regionales, como locales como la aplicación del plan maestro de transporte que regula la circulación y mantención de los vehículos, debido a que fuentes móviles aportan $48 \%$ de las toneladas de $\mathrm{MP}_{10}$ de la región ${ }^{21}$. Fortalecer la aplicación de planes de reforestación, que contempla a Cerro Navia dentro de las comunas priorizadas para creación de áreas verdes, como parte del plan de descontaminación de la región ${ }^{22}$. Además, si este aumento de carga de enfermedad respiratoria se mantuviera en el tiempo, se requeriría una com-

\section{REFERENCIAS}

1. Epa's Science Advisory Board: The EPA'S particulate matter (PM). Health effects research centers program. USA Report Issued Jan 2002.

2. Ostro B, Sánchez JM, Aranda C, Eskeland GS. Air pollution and mortality: results from a study of Santiago, Chile. J Expo Anal Environ Epidemiol 1996; 6: 97-114.

3. Ostro B, Eskeland GS, Sánchez JM, Feyzioglu T. Air pollution and health effects: A study of medical visits among children in Santiago, Chile. Environ Health Perspect 1999; 107: 69-73.

4. Ilabaca M, Olaeta I, Campos E, Villaire J, TellezRojo MM, Romieu I. Association between levels of fine particulate and emergency visits for pneumonia and other respiratory illnesses among children in Santiago, Chile. J Air Waste Manag Assoc 1999; 49 (9 Spec No): 154-63. pensación en el financiamiento para la atención de salud, que podría ser el incremento del per cápita comunal asociado al mayor riesgo de la población.

En resumen, nuestro estudio muestra que la morbilidad respiratoria en el CA tiene un claro componente estacional, similar a la $\mathrm{RM}$ en su conjunto y que la proporción de consultas de morbilidad respiratoria en niños por SBO y neumonía son significativamente mayores en CA de Cerro Navia en comparación con la RM y que, en el adulto mayor, la enfermedad respiratoria de la vía aérea inferior, también es mayor en el CA. Todos estos hallazgos podrían estar relacionados con las concentraciones elevadas de $\mathrm{MP}_{10}$, lo que tendría que corroborarse con nuevos estudios. Dado que no se han realizado hasta la fecha estudios de contaminación y efectos en salud respiratoria a nivel comunal con medición de consultas diarias en Atención Primaria, esta aproximación descriptiva puede dar lugar a nuevas investigaciones que permitan a través de promedios móviles, y análisis de regresión múltiple, aislar las diferentes variables para determinar el peso de los contaminantes en la morbilidad respiratoria a nivel local.

5. ARANDa C, Astudiwo P. Comelación entre contaminación por $\mathrm{PM}_{10}$ y mortalidad por neumonía en niños de Santiago. Rev Chil Enf Respir 1991; 7(S): 33.

6. Román O, Mancila P, Prieto MJ. Contaminación atmosférica y daño cardiovascular. Rev Méd Chile 2004; 132: 761-7.

7. Gil L, Martínez V, Riquelme R, Ancic P, González G, RodRíGUEz L ET AL. Occupational and environmental levels of mutagenic PAHs and respirable particulate matter associated with diesel exhaust in Santiago, Chile. Journal of Occupational and Environmental Medicine 2003; 45: 984-92.

8. Rosaies Ja, Torres VM, Oiazz G, Borja VH. Los efectos agudos de la contaminación del aire en la salud de la población: Evidencias de estudios epidemiológicos. Salud Pública Mex 2001; 43: 544-55.

9. Lazcano F, Westhof D, Lima J. Sitios centinela, monitoreo y evaluación. UNICEF CA. 1991; Sept. Mimeo, p 1-8. 
10. Aranda C, Astudilo P, Mancilla P, Caussade S, Girardi G, Gamboa R. Monitoreo epidemiológico de los efectos de la contaminación atmosférica en las enfermedades respiratorias infantiles. 1992; Dic Mimeo, UNICEF.

11. Olaeta I. Métodos de monitoreo de la calidad del aire en la Región Metropolitana (Red MACAM). Rev Chil Enf Respir 1991; 7: 186-90.

12. Ministerio de Salud Chile, SEREMI RM, Sección Vigilancia Calidad del Aire 2004.

13. Ministerio de Salud Chile. Norma Técnica Manejo de las Enfermedades Respiratorias del niño, Atención Primaria de Salud 2002.

14. Ministerio de Salud Chile. Norma Técnica Programa de control de las Enfermedades Respiratorias del Adulto en Chile, resolución exenta no 2282 año 2002.

15. Cáceres L, Adonis M, Retamal C, Ancic P, Valencia $\mathrm{M}$, RAmos X et AL. Contaminación intradomiciliaria en un sector de extrema pobreza de la comuna de La Pintana. Rev Méd Chile 2001; 129: 33-42.

16. Aranda C, Astudilo P, Mancila P, Caussade S, GIRARDI G. Caracterización epidemiológica de las consultas pediátricas por causa respiratoria en atención primaria en Chile. OPS serie HCT/ AIEPI-3.E 1998; Dic p 35-42.

17. Donoso E. Desigualdad en mortalidad infantil entre las comunas de la provincia de Santiago. Rev Méd Chile 2004; 132: 461-6.

18. SERPLAC R.M. «Región Metropolitana de Santiago. Evolución Indicadores Sociales 1990-2003: Comparación Resultados Encuestas CASEN», №3 Colección «Ciudad y Territorio», Junio 2005.

19. CONAMA Distribución de contaminantes atmosféricos en RM Informe 2003.

20. CONAMA: «Mejoramiento del Inventario de Emisiones de la Región Metropolitana» Diciembre 2000 Santiago, Chile.

21. Brote de Influenza y VRS en la Región Metropolitana, Invierno 2004. Disponible en: http:// epi.minsal.cl/evigant/Numero27/ evigia/html/portada/centro.htm (Acceso el 19 de mayo de 2006)

22. Diario Oficial: Modificación de DS 16 de 1998, del Ministerio Secretaría General de la Presidencia, que establece el Plan de Prevención y Descontaminación Atmosférica de la Región Metropolitana Núm. 20.- Santiago, 22 de enero de 2001. 